\title{
Average crack-front velocity during subcritical fracture propagation in a heterogeneous medium
}

\author{
Olivier Lengliné, Renaud Toussaint, and Jean Schmittbuhl \\ IPGS, EOST, CNRS, Université de Strasbourg, 5 rue René Descartes, F-67084, Strasbourg, France \\ Jean E. Elkhoury and J. P. Ampuero \\ Seismological Laboratory, California Institute of Technology, Pasadena, CA 91125, USA \\ Ken Tore Tallakstad, Stéphane Santucci, ${ }^{*}$ and Knut Jørgen Måløy \\ Department of Physics, University of Oslo, PB 1048 Blindern, NO-0316 Oslo, Norway \\ (Received 16 February 2011; revised manuscript received 15 June 2011; published 9 September 2011)
}

\begin{abstract}
We study the average velocity of crack fronts during stable interfacial fracture experiments in a heterogeneous quasibrittle material under constant loading rates and during long relaxation tests. The transparency of the material (polymethylmethacrylate) allows continuous tracking of the front position and relation of its evolution to the energy release rate. Despite significant velocity fluctuations at local scales, we show that a model of independent thermally activated sites successfully reproduces the large-scale behavior of the crack front for several loading conditions.
\end{abstract}

DOI: 10.1103/PhysRevE.84.036104

PACS number(s): 46.50.+a, 62.20.M-

\section{INTRODUCTION}

The mechanisms responsible for the onset and evolution of fracture propagation are of central importance for the assessment of failures in man-made structures and natural materials such as rocks, sea ice, and wood [1-4]. In the general context of fracture mechanics, several regimes of behavior are often reported. According to the Griffith energy balance concept, in a quasistatic regime, the energy release rate $G$ is roughly constant and equal to a material-dependent critical energy release rate, $G \sim G_{g r}$ [5]. $G_{g r}$ is the free energy per unit surface area associated with the creation of new crack surfaces. The kinetic energy becomes important in unstable configurations or during fast loading leading to a dynamic regime [6,7]. In the slow, unstable regime (i.e., rupture velocity smaller than the Rayleigh wave speed), the crack propagation speed is roughly proportional to the difference between $G$ and $G_{g r}$ (e.g., [8]) and strongly controlled by loading conditions (imposed stress or imposed displacement, loading rate) and material rheology. Modeling approaches in these cases are typically based on the linear elastic fracture mechanics (LEFM) approximation [9].

A generalization of the Griffith concept of fracturing, in the framework of irreversible processes, allows for the propagation of fractures at speeds below that of the Rayleigh wave for energy release rate lower than $G_{g r}$ [10]. In consequence the slow kinetic crack propagation is usually referred to as subcritical crack growth or the subcritical regime. Statistical physics models suggest that this subcritical regime is governed by a thermally activated mechanism where the strain rate often obeys an Arrhenius law, i.e., corresponds to a Boltzmannian of the gap to a critical energy level $G_{c}-G[5,11,12]$. The crack growth is directly influenced by environmental factors (applied stress, temperature, chemical concentrations) affecting the free

\footnotetext{
*Present address: Laboratoire de Physique, CNRS UMR 5672, Ecole Normale Supérieure de Lyon, 46 allée d'Italie, 69364 Lyon Cedex 07, France.
}

energy, and thus the energy barrier, via numerous competing mechanisms like stress corrosion, diffusion, dissolution, and plasticity [1,5]. Several experimental empirical relations have been reported that divide slow crack propagation into three main characteristic regimes depending on the crack speed [13]. These regimes reflect the competition between crack growth velocity and diffusion of species at the crack tip. At very slow velocity, external variables are dominant and an increase in $G$ results in an increase in the speed of crack growth. A weak stress-sensitive regime follows where transport is limited. At higher stress, the crack growth kinetics becomes similar to that in the case with no environmental effects.

Other relations besides the Arrhenius law have also been reported to describe slow crack growth. These relations are generally inferred from fitting an empirical relation to experimental data on a $G-\bar{v}$ diagram, where $\bar{v}$ is the crack velocity. Such relations involve power laws with small exponents [14], or large exponents when the crack propagation is more sensitive to chemical reaction rates $[15,16]$. To simplify the relationship between strain rate (i.e., crack-front advance) and stress, some models consider the crack propagation speed as roughly proportional to the difference between $G$ and an energy release rate threshold, or to a power law of this difference, i.e., a Paris law (e.g., [17-19]).

The Arrhenius form of the kinetic fracture evolution is appealing as it has a certain universality associated with statistical mechanics. It is related to any thermally activated mechanism at the molecular scale and can be derived from first principles as the theory of process rates [5]. Such a relation is also supported by experimental data $[1,20]$, and if the dependence of the free energy $G$ on the strain can be adequately linearized, a logarithmic time dependence of the strain, and force, can be derived. This is observed for many systems under creep like granular systems displaying a temporal logarithmic deformation (or force) during slow relaxation [21-23].

For heterogeneous materials, crack propagation is, however, influenced by local variations of the material properties, microstructures, thermal fluctuations, and the development of 
a process zone [24]. The interplay of the different contributions to the crack growth makes its analysis a complex problem. It is indeed difficult to discriminate the part of each contribution by observing the evolution of a crack advance at a microscopic level. The failure of brittle materials with weak flaws is typically described by Weibull statistics of the flaw strengths which relies on the weakest-link approach and extreme-value statistics [25]. Quasibrittle materials exhibit a departure from the Weibull statistics for which ductile behavior around the crack tip imposes an representative elementary volume (REV) of non-negligible size compared to the sample size. These materials show significant scaling or size effects, in particular at very low loading rates [26].

The interfacial crack propagation configuration has long been a favorable experimental setting for fine monitoring of crack propagation. It consists of a single cantilever beam configuration with fracture propagation along a weak interface. It was the first setup to enable measurements of fracture energy in brittle materials like mica [27]. In the case of quasibrittle materials [e.g., polymethylmethacrylate) (PMMA)], it provided the first detailed observation of the morphology of the crack front $[28,29]$. The distribution of local velocities of the crack front displays an intermittent behavior at small scales [30,31]. The scaling properties of the crack front remain unchanged in both the high and slow speed limits at the local dynamic [31]. This suggests that a common mechanism might be operating in these two regimes. This was the case for both the front's geometrical properties (scaling of the front roughness) and the time and space scaling properties of the velocity fluctuations. The interfacial configuration is also favorable for numerical simulations since it reduces the roughening of the crack front to an in-plane problem. Two modeling approaches have been proposed: (1) a microscopic description of the crack front as a continuous line [8,32-34] and (2) fiber bundle models in which a discrete population of active sites are competing under various interaction rules [35-39].

Here, we focus on the experimental description of the spatially averaged velocity of the crack front in the interfacial fracture configuration, under various loading conditions. Our heterogeneous medium consists of an interface between two PMMA plates with small toughness fluctuations [40]. Each local asperity is a site of a depinning transition that leads to a progressive local advance of the crack front. We optically monitor the global evolution of the crack by taking advantage of the transparency of our sample. We explore the regimes of small loading rates (up to $1 \mathrm{~mm} / \mathrm{s}$ ) and relaxation at fixed load point displacement. First, we compare the average crack velocity to the loading velocity or the energy release rate for both loading regimes. Then we show that the large-scale evolution of the crack-front velocity can be explained by a simple model of a population of statistically independent but temperature-sensitive microscopic active sites, slowly evolving, following an Arrhenius law. Numerical solutions of the model correctly describe the experimental behaviors for time scales spanning over six orders of magnitude for both loading types. The parameters of the subcritical crack-growth law provide a characteristic size of the individual breaking bond. For our case, we obtain a length scale of $10^{-11} \mathrm{~m}$ for PMMA comparable to the separation of individual molecular elements and much smaller than the scaling of the fracture front $\left(10^{-5}\right.$ to $\left.10^{-2} \mathrm{~m}\right)$ similar to the scales determined for granite [41], paper [42], and numerous other materials [12].

In addition to the numerical solutions, we derive simple analytical expressions for the two loading regimes under several approximations. Notably, we find a slow timelogarithmic relaxation. We also show that $G \sim G_{g r}$, for imposed loading rates, results from an Arrhenius law with stress-activated microscopic sites and linear elasticity that describe the dependence of the stress on the large-scale geometry.

\section{EXPERIMENTAL ANALYSIS}

\section{A. Sample preparation}

We use two PMMA plates of dimensions $20 \times 10 \times$ $1.0 \mathrm{~cm}^{3}$ and $23 \times 2.7 \times \mathrm{cm}^{3}$. First, we sandblast one surface of the narrower plate with glass beads of diameter $\phi(\phi \in$ [180-300] $\mu \mathrm{m})$. The blasted plate is cleaned to remove any electrically attached glass beads. Then, we assemble the two plates in a stiff aluminum frame with the blasted surface facing a surface of the larger plate. Finally, we subject the assembled plates to a homogeneous normal load of $3 \mathrm{MPa}$ and heat the assembly in an oven at $190{ }^{\circ} \mathrm{C}$ for $45 \mathrm{~min}$ to anneal the plates. The thermal annealing produces a cohesive interface that is weaker than the bulk and constrains the sample to break along the prescribed interface. The glass bead blasting introduces random heterogeneities in the surface topography that provides surface roughness and controls the local strength at the interface. The induced microstructure at the interface makes the sample opaque. However, the newly formed block, after annealing, recovers its transparency since contrast of the refractive index along the interface disappears.

\section{B. Acquisition and image processing}

We clamp the large plate of the fully healed sample to a stiff aluminum frame. A stepping motor applies the loading to the tip of the narrow plate normal to the plate surface (Fig. 1). The contact on the plate is imposed by a freely rotating rod (using ball bearings), made of low-friction-coefficient material (polyamide PA 6.6). We measure the vertical displacement at the loading point using a linear variable differential transformer (LVDT) and the force load by an STC 1205 tractioncompression transducer. Displacement and force are measured to a resolution of $1.3 \mu \mathrm{m}$ and $2.4 \times 10^{-3} \mathrm{~N}$, respectively. The vertical displacement imposed on the narrower sample induces the stable propagation of a planar fracture along the prescribed interface. The loading velocity varies for each experiment within the range [6-600] $\mu \mathrm{m} / \mathrm{s}$ and is zero during creep tests. This leads to null front velocities at the start of experiments and maximum front velocities on the order of $3.0 \times 10^{3} \mu \mathrm{m} / \mathrm{s}$. The total advance of the crack front achieved during an experiment is typically on the order of $1.0 \mathrm{~cm}$.

We monitor the fracture front optically using a Nikon D700 camera with up to $\sim 5$ frames per second to follow the crack front propagation (Fig. 1). The optical images are $4256 \times 2832$ pixels and of a resolution in the range [5-10] $\mu \mathrm{m} /$ pixel. The optical images of the interfacial rupture are divided into bright and dark regions representing the cracked open and unbroken sections of the sample respectively. To obtain the 


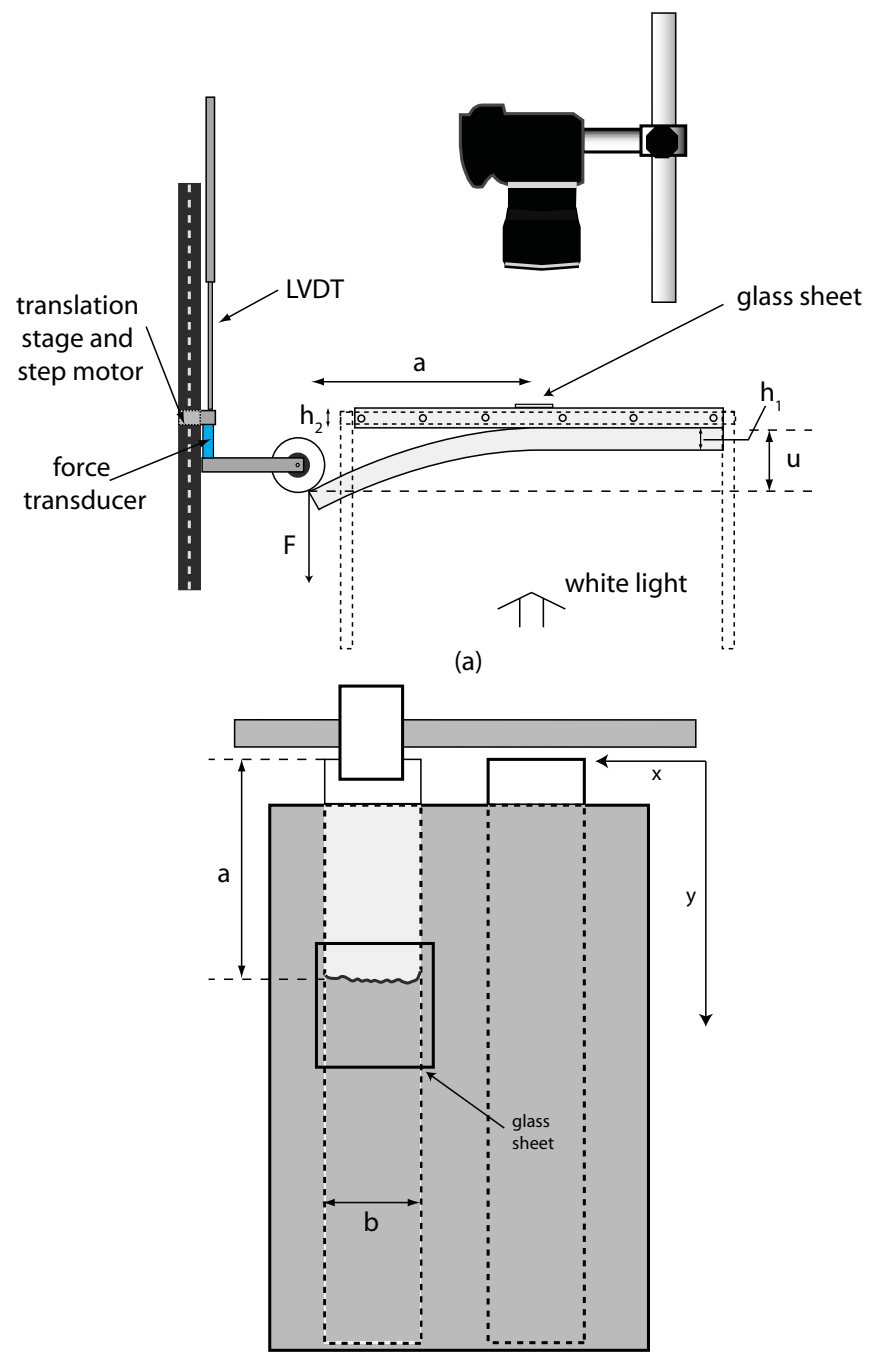

(b)

FIG. 1. Side view (top) and top view (bottom) of the experimental setup. A stiff aluminum frame is attached to the upper PMMA plate. The lower plate is separated from the upper one by a load $F$ applied by a rod connected to a stepping motor. The rod can freely rotate around its axis. The load causes a deflection $u$ of the bottom plate and the propagation of an interfacial crack. The crack front is located at the average distance $\bar{a}$ from the free end ( $\bar{a}$ is obtained as the spatial average of the distance $a$ along the front). Front advance is monitored by a camera set in vertical position, perpendicular to the crack plane. We add glycerol between the upper plate and a thin glass sheet located above the crack front to enhance the optical contrast of the pictures. The narrow plate thickness and width are denoted $h_{1}$ and $b$, respectively, while the large plate thickness is denoted $h_{2}$.

transition between the bright and dark regions that represents the fracture front, we first compute the difference of the grayscale maps between each image and the first image of the experiment. This first step highlights the difference between two consecutive time stamps of the fracture process while removing permanent artifacts associated with index variations in the material. Then, we transform the grayscale images to black and white according to a threshold that represents the gray level separation between the bright and dark regions. The fracture front $a(x, t)$ is the largest cluster of connected pixels with nonzero gradient. The front propagates on average along the $y$ axis where the origin is defined at the free end of the plate and is positive in the direction of propagation. The $x$ axis is perpendicular to the $y$ axis and defines the coordinate of a point along the front, $t$ is time, and $a(x, t)$ is the $y$ position of the front at lateral position $x$ and time $t$. We compute the average front position $\bar{a}(t)$ from each profile $a(x, t)$ [30,40].

\section{Typical run of an experiment}

All experiments start with no initial deflection, i.e., the loading point is not in contact with the narrow PMMA plate. Then the stepping motor moves the loading point at controlled speed $V_{l}$. Once contact is achieved between the loading point and the plate, both the force $F$ at the free end of the plate and the deflection $u$ increase. The force increase leads to the initiation of crack movement while the loading point continues to move at constant velocity. The duration of this constantloading-velocity regime is inversely proportional to $V_{l}$. The fracture front speed decreases once we stop the stepping motor ( $V_{l}=0$, at $t=t_{\text {stop }}$ ). We typically monitor the evolution of the fracture fronts for about $5 \mathrm{~min}$. Monitoring lasted for more than $18 \mathrm{~h}$ for one of our experiments. We unload the sample by moving the loading point backward to its initial position, leading to the arrest of the front. The next experiment starts where the previous one ends until the front reaches the end of the plate. We performed all experiments at room temperature ( $T \in[22.2-24.4]^{\circ} \mathrm{C}$ ) and used two completely independent experimental setups run by different groups, with no noticeable discrepancies in the results.

An example of typical experimental measurements is shown in Fig. 2. Here, the sample was continuously loaded at $62 \mu \mathrm{m} / \mathrm{s}$ during $230 \mathrm{~s}$. Rupture initiates at $t \sim 190 \mathrm{~s}$, as evidenced by the onset of the front movement and the deviation of the force from the linear trend. We observe an increase in the crack velocity as long as the sample is continuously loaded. We then maintain the loading point at a fixed position at time $t_{\text {stop }}=230 \mathrm{~s}$ as indicated by the vertical gray line (Fig. 2). The front velocity exhibits a deceleration while the force is decreasing when the loading point velocity is set to zero. We performed all the experiments following the same scheme presented in Fig. 2.

\section{EXPERIMENTAL OBSERVATIONS}

In the following subsections we report on the behavior of the average front positions with time in the two loading regimes, at constant loading velocity, or at constant loading position, and show that they follow simple laws. In subsequent sections, we derive these laws on a theoretical basis and interpret their parameters in a physical context.

\section{A. Constant loading velocity}

In the constant-loading-velocity regime, we observe an initiation phase which precedes the propagation of the crack at an almost constant velocity (Fig. 2). The evolution of the front position $\bar{a}$ as a function of the loading point displacement $u$ is shown in Fig. 3. We only show data for $t<t_{\text {stop }}$ where the dynamics of the crack is driven by the applied load at constant velocity. We observe that the evolution of $\bar{a}$ is well approximated by a fit of the form $\bar{a}(u) \propto \sqrt{u}$ (dashed lines in Fig. 3) for each of our samples. 


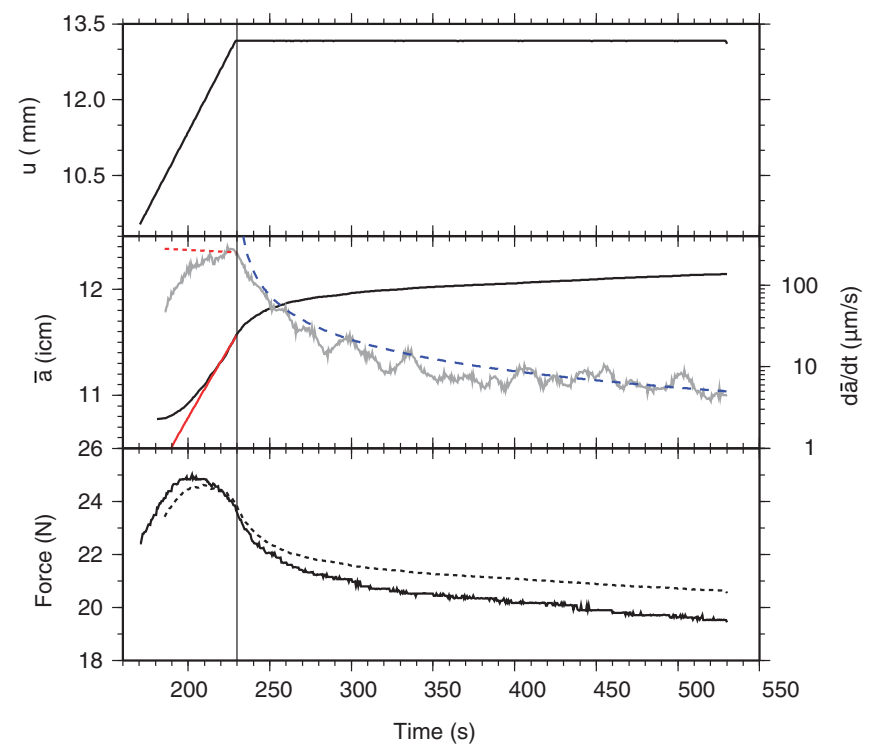

FIG. 2. (Color online) Top: Variation of loading point position $u$ as a function of time during an experiment. At time $t=230 \mathrm{~s}$, the loading point is maintained in a fixed position. Middle: Variation of the average front position $\bar{a}$ as a function of time (black solid line) and evolution of the crack front velocity $d \bar{a} / d t$ (gray solid line). The red solid and short-dashed lines represent a fit to the average front position and velocity, respectively, according to Eq. (19). The blue long-dashed line is a fit to the crack velocity in the relaxation regime following Eq. (26). Bottom: Variation of force as a function of time (black solid line) and force predicted by the beam theory (gray dashed line) using (5) with $E=3.2 \mathrm{GPa}$ and $b=2.84 \mathrm{~cm}$, which are the measured properties of the plate. For all figures, the vertical gray line denotes the time at which the loading was stopped and separates the imposed velocity regime from the relaxation regime.

\section{B. Constant-deflection condition}

Now we turn to the relaxation regime characterized by a constant deflection $u\left(t>t_{\text {stop }}\right)$. We observe a progressive deceleration of the front (Fig. 2). In order to precisely describe the evolution of the front position in this particular regime, we set $\bar{a}_{0}=\bar{a}\left(t_{\text {stop }}\right)$ and we represent the evolution of the front position $\bar{a}(t)-\bar{a}_{0}$ for time since $t_{\text {stop }}$. We observe a logarithmic evolution of the front advance for large times (Fig. 4). We propose to fit the advance of the front by a logarithmic relation of the form

$$
\bar{a}\left(t-t_{\text {stop }}\right)-\bar{a}_{0}=A \ln \left(\frac{t-t_{\text {stop }}}{t^{*}}+1\right),
$$

where $t^{*}$ is a characteristic time and $A$ an empirical constant. Equation (1) provides a good fit (Fig. 4). We obtain typical values of $t^{*}$ on the order of $1-10 \mathrm{~s}$, while typical values of $A$ are on the order of $10^{-4}-10^{-3} \mathrm{~m}$. We also report the evolution of the front position during this relaxation regime for an experiment lasting more than $18 \mathrm{~h}$ (Fig. 4). The propagation of the front remained small $(\sim 2 \mathrm{~cm})$ even for this long-term experiment. The logarithmic evolution of the crack front contrasts with the previous regime of constant loading velocity. The large-scale progression of the front must be dominated by a process capable of capturing such distinct behaviors.

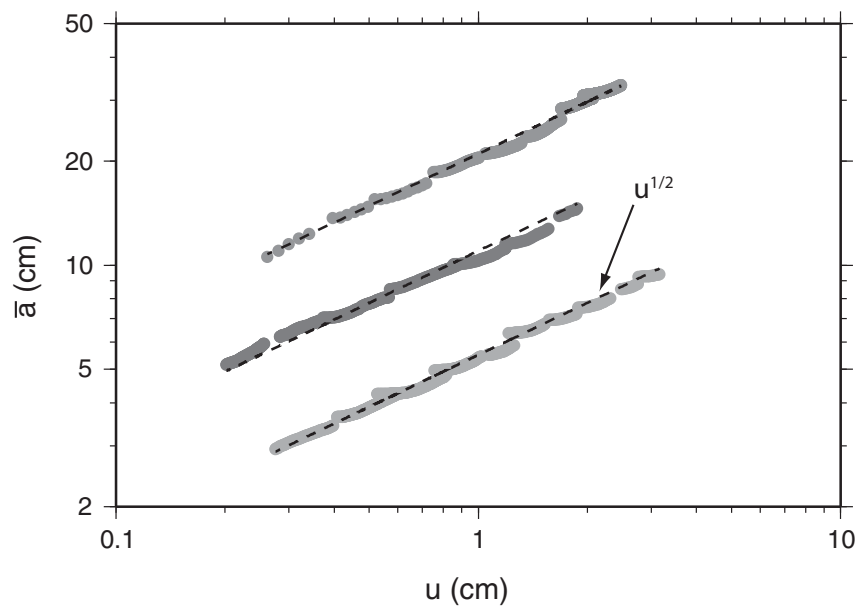

FIG. 3. Variation of the crack front position $\bar{a}$ as a function of the loading point displacement $u$. The gray points refer to recorded data from different samples. For each sample we carried out several experiments. The best fit using $\bar{a}(u) \propto u^{1 / 2}$ is displayed as a dashed line for each sample. Crack-front positions are shifted vertically for each sample in order to enhance the visibility (by a prefactor corresponding respectively to a factor 0.5 for the lowest and 2 for the highest curves). We see that for each sample and for each experiment the observed behavior is in good agreement with the fitted trend. Notice, however, that the crack initiation does not seem well approximated by this trend.

\section{SUBCRITICAL CRACK PROPAGATION MODEL}

\section{A. Thermally activated microscopic process}

We assume that the crack propagation is governed, at the local scale, by a subcritical mechanism based on an Arrhenius law [14]. Owing to thermal fluctuations large enough to overcome the fracture energy barrier of individual bonds, the fracture propagates at slow speed. This contrasts with the Griffith approach where no propagation is allowed below the Griffith energy release rate $G_{g r}$ (or below the Griffith stress intensity factor $K_{g r}$ following an Irwin criterion [5]). The fracture energy barrier fluctuates in space but it is constant at a characteristic microscopic scale $\alpha$, which is a length scale associated with individual degrees of freedom of the microscopic fracturing process. At zero loading, we consider that the magnitude of the barrier is related to the local critical energy release rate $\alpha^{2} G_{c}$. This aspect of a quenched fluctuating zero-loading barrier relates material properties to $G_{c}$. The energy gap is also dependent on the elastic mechanical energy around the crack tip which is described by the local energy release rate $\alpha^{2} G$. Such models of coupled thermal noise and spatial disorder have been previously investigated in some theoretical approaches of fiber bundle models and irreversible crack growth $[43,44]$. The probability for an individual asperity to go beyond the barrier $\Delta G=G_{c}-G$ per period characteristic of the microscopic thermal motion $\tau_{B}$ is the Boltzmannian of the relative elastic energy $\alpha^{2} \Delta G$. The Arrhenius relation governing the local fracture velocity $v=d a / d t$ can thus be written as

$$
v\left(G, G_{c}\right)=\psi \exp \left(-\alpha^{2} \Delta G / k_{B} T\right)
$$



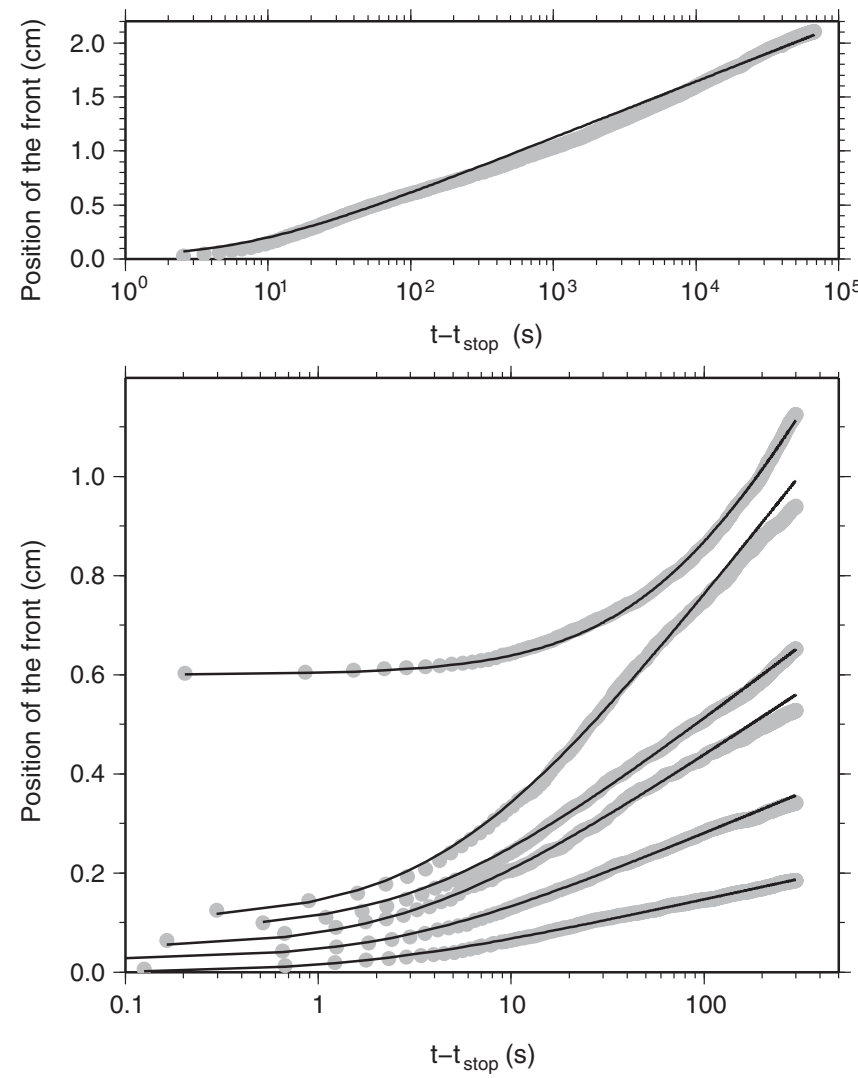

FIG. 4. Evolution of the average front position $\bar{a}(t)-\bar{a}_{0}$ during several relaxation experiments as a function of time $t-t_{\text {stop. The }}$ observed front position is represented by gray dots while the best fits using (1) are displayed as continuous dark lines. Traces have been shifted vertically in order to enhance visibility. The experiment displayed on the top figure lasted more than $18 \mathrm{~h}$ (the time representation scale is different from the one in the bottom figure).

where $\psi$ is a constant of proportionality equal to $\alpha / \tau_{B}, T$ is the absolute temperature (in kelvin), and $k_{B}$ is the Boltzmann constant.

\section{B. Population of independent microscopic sites}

We propose a model for the average crack velocity based on a statistical approach. The behavior of the weak interface is assumed to be dominated at large scales by a discrete set of independent microscopic sites. We obtain the largescale evolution law of the crack-front speed by integrating Eq. (2) over all local microscopic sites at a given time. Such an integration is not straightforward as there might exist correlations among local values of $G$ and $G_{c}$. Fluctuations in $G$ results from the crack front roughening around its average position $\bar{a}$ [32] and a perpendicular, large-scale, stress gradient due to bending of the loading plate. For the fracture energy $G_{c}$, the quenched fluctuations result from the sample preparation. Such fluctuations introduce correlation over a finite length of local velocities [31]. We hypothesize that at the macroscopic scale, above these correlation lengths, the average (or large-scale) evolution of the front is governed by a law similar to Eq. (2) but involving only large-scale average quantities. $\bar{G}$ describes the average loading along the front and $\bar{G}_{c}$ describes the upscaled energy barrier at the front scale.
The critical size of the degree of freedom, $\alpha$, is preserved. For example, in a simple model where all parameters were statistically independent and $G$ and $G_{c}$ could be described by two independent normal distributions with respective means $\bar{G}$ and $\bar{G}_{c}$ and root mean squares $\sigma_{G}$ and $\sigma_{G_{c}}$, we would get

$$
\bar{v}=\chi \exp \left[-\alpha^{2}\left(\bar{G}_{c}-\bar{G}\right) / k_{B} T\right],
$$

where

$$
\chi=\psi \exp \left[\frac{\alpha^{4}\left(\sigma_{G}^{2}+\sigma_{G_{c}}^{2}\right)}{2 k_{B}^{2} T^{2}}\right]
$$

We acknowledge that, at small scales, $G$ and $G_{c}$ are not statistically independent and that intermittent behavior, large fluctuations of velocity, and spatial correlations in $G$ and in $G_{c}$ have been observed and described in detail (e.g., $[30,31])$. Nonetheless, we assume that the upscaling leads to a simple form as in Eq. (3). The upscaling could result from a discretization scale above which asperities could be considered independent. This discretization scale could be analogous to the REV introduced by Bažant and Pang [45] for quasibrittle materials. Within each REV, the effective failure of the asperity is considered as a purely thermally activated and stressdependent process. This model is not continuous in contrast to the contact line approach [8,32-34]. It can rather be compared to fiber bundle models [35-39] or interacting damage models [46-49] but with a nondirect stress redistribution.

For simplicity, we ignore mechanical interactions among microscopic sites in describing the dynamics of the upscaled spatially averaged velocity. Only the mechanical energy release rate, controlling the upscaled velocity, depends on the large-scale average front position. The upscaling of Eq. (2) leads to a similar form with only upscaled energy release rates and energy barrier [Eq. (3)]. This assumption will be checked by comparing this simple model to our experimental observations. Below we show that the model holds for the relaxation regime and also for the forced regime.

\section{A cantilever configuration}

We estimate the average energy release rate $\bar{G}$ using classical elastic plate theory, neglecting fluctuations of the front position around an average front position $\bar{a}$. The average energy release rate from the elastic strain energy, $U_{E}$, stored in the loading plate is

$$
\bar{G}=-\frac{1}{b} \frac{d U_{E}}{d \bar{a}},
$$

where $b$ is the width of the plate [5]. The linear relation between the applied force $F$ and the deflection of the beam $u$ from one-dimensional (1D) beam theory is

$$
F=\frac{u E b h^{3}}{4 \bar{a}^{3}},
$$

where $E$ is the Young modulus of the narrow plate and $1 / h^{3}=$ $1 / h_{1}^{3}+1 / h_{2}^{3}$ is an equivalent thickness resulting from our asymmetric configuration (see [5] and details in the Appendix, where we also estimate both mode I and mode II loading). This leads to the following expression of the elastic energy:

$$
U_{E}=F \frac{u}{2}
$$


Combination of Eqs. (4), (5), and (6) gives an estimation of the average energy release rate in our system,

$$
\bar{G}=\frac{3 u^{2} E h^{3}}{8 \bar{a}^{4}} .
$$

\section{Macroscopic evolution law}

We derive the expression for the evolution of the crack-front velocity by combining Eqs. (3) and (7),

$$
\bar{v}=\frac{d \bar{a}}{d t}=\chi \exp \left(\frac{\alpha^{2}}{k_{B} T}\left[\frac{3 u^{2} E h^{3}}{8 \bar{a}^{4}}-\bar{G}_{c}\right]\right) .
$$

Equation (8) is the differential equation that governs the motion of the front in our experimental configuration. This equation takes different forms depending on the loading scheme, which manifest in the expression of the time-dependent imposed deflection $u(t)$. We explored two particular loading conditions imposed on the experimental system, namely, a constantloading-velocity condition and a fixed-deflection condition.

As a first check, we computed the average crack-front velocity $\bar{v}(t)$. We smoothed $\bar{a}(t)$ values with a window running along time and then dividing each incremental position by the time interval between successive images. We estimated $\bar{G}$ at each time interval from Eq. (7). The average crack-front velocity is shown in Fig. 5 as a function of the average energy release rate $\bar{G}$ using Eq. (7). We clearly observe that

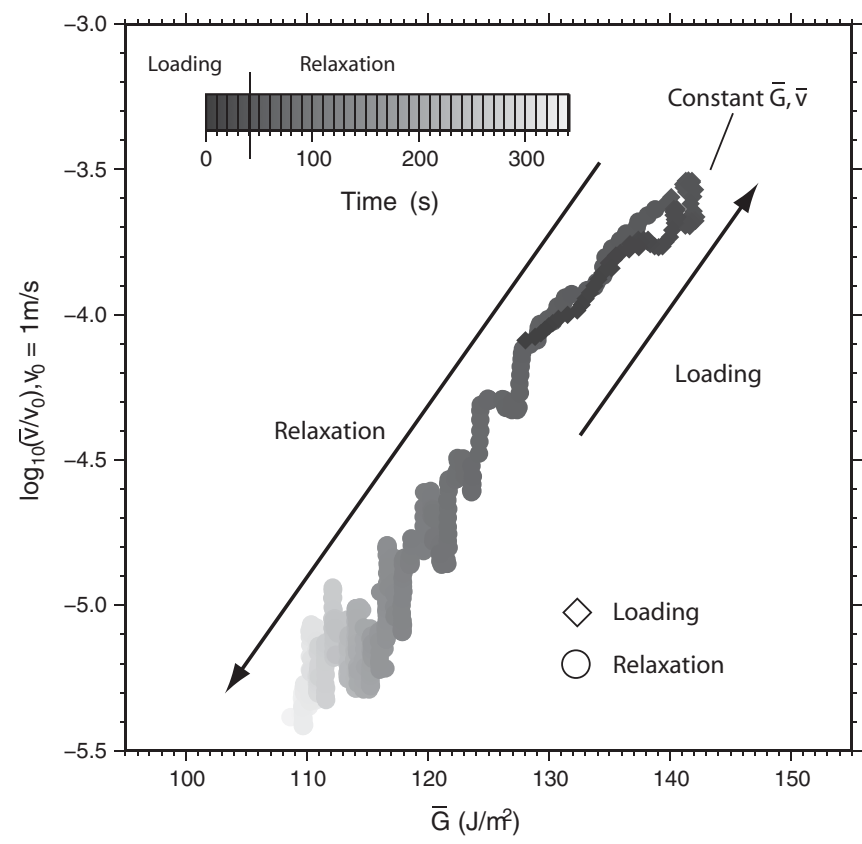

FIG. 5. Variation of the logarithm of the average crack-front velocity as a function of the average energy release rate during an experiment. The grayscale refers to time since the picture acquisition starts. Both loading regimes (constant loading velocity, diamonds, and relaxation regimes, circles) are included. We see the increase of $\bar{G}$ and $\bar{v}$ during the constant-loading-velocity regime, the stabilization of $\bar{G}$ while the front is propagating at almost constant speed, and the decrease of $\bar{G}$ and $\bar{v}$ during the relaxation process. The linear relationship between $\bar{G}$ and $\log _{10}(v)$ is observed during all these phases. a linear relationship $[\ln (\bar{v})$ vs $\bar{G}]$ is a reasonable proxy of the complete behavior of the experiment where the sample undergoes multiple loading cycles: first a constant-loadingvelocity condition followed by a constant-deflection condition. We also notice that $\bar{G}$ is almost constant while the front velocity $\bar{v}$ reaches a nearly steady value. The linear relationship $[\ln (\bar{v})$ vs $\bar{G}]$ is valid for all 13 experiments shown in Fig. 6. The horizontal shift in $\bar{G}$ between experiments results from variations of the fracture energy $\bar{G}_{c}$ from plate to plate. Large-scale variations of the fracture energy on a given plate are also expected. It results from the annealing process during sample preparation, since temperature, plate thickness, and normal load applied on the sample are not perfectly uniform and may vary among plates [50]. As $\bar{G}_{c}$ cannot be solely estimated from our experimental results (due to its coupling with $\chi$ ), we set an arbitrary characteristic reference velocity in our experiments $v_{\text {ref }}$ and searched for an estimate of a reference energy release rate $\bar{G}^{\text {ref }}=\bar{G}\left(v_{\text {ref }}\right)$ for each experiment that provides the best superposition of all experimental curves [see Fig. 6(b)]. Following Eq. (3), we get

$$
\ln \left(\bar{v} / v_{\text {ref }}\right)=\frac{\alpha^{2}\left(\bar{G}-\bar{G}^{\text {ref }}\right)}{k_{B} T}
$$

for each experiment. We set the reference velocity to $10^{-4}$ $\mathrm{m} / \mathrm{s}$, which is a typical order of magnitude of the crack-front velocity in our experiments. Although values of $\bar{G}^{\text {ref }}$ depend on the particular choice of $v_{\text {ref }}$, the variation of $\bar{G}^{\text {ref }}$ among experiments remained constant regardless of the choice of $v_{\text {ref }}$. We report values of $\bar{G}^{\text {ref }}$ in Fig. 7. We used $k_{B}=1.38 \times$ $10^{-23} \mathrm{~J} / \mathrm{K}$ and $T=300 \mathrm{~K}$ and obtained the value of $\alpha$ by least squares fitting over all experiments: $\alpha=2.5 \times 10^{-11} \mathrm{~m}$. $\alpha$ is the characteristic length scale at which the elementary fracturing process is expected to occur. This implies that the rupture process is governed by mechanisms operating at the scale of the bond distance between atoms. This is consistent with the underlying physics of the Arrhenius law, where each individual degree of freedom in the system can break with a certain probability set by thermodynamics to be a Boltzmann distribution. These individual degrees of freedom probably correspond to individual molecules crossing through the weak interface.

The estimated reference energy release rate $\bar{G}^{\text {ref }}$ has a median and mean of 133 and $137 \mathrm{~J} / \mathrm{m}^{2}$ respectively with $20 \%$ fluctuations (Fig. 7). Such a range of variation is compatible with sample preparation variability (plate thickness fluctuation, non-fully-homogeneous annealing procedure, sandblasting variability, etc.) and is also consistent with results obtained in [50]. Furthermore, variations of $\bar{G}^{\text {ref }}$ are found to be small for experiments that are performed on the same PMMA plate and close to each other (i.e., consecutive experiments that were initiated just one after the other on a small region of the plate) but with significant differences when changing plates or exploring very different zones of the same plate [Fig. 6(a)]. It therefore suggests that the horizontal shift observed on Fig. 6(a) between experiments results from variations in $\bar{G}_{c}$.

Other relations linking the crack speed to the energy release rate have been proposed. These are (1) $\bar{v} \sim e^{-E^{\prime} k_{B} T} G^{n / 2}$ [15] and (2) transforming $\Delta G$ in (3) into $\Delta G^{-\mu}$, where the exponent $\mu$ arises from possible 3D paths taken by the crack 

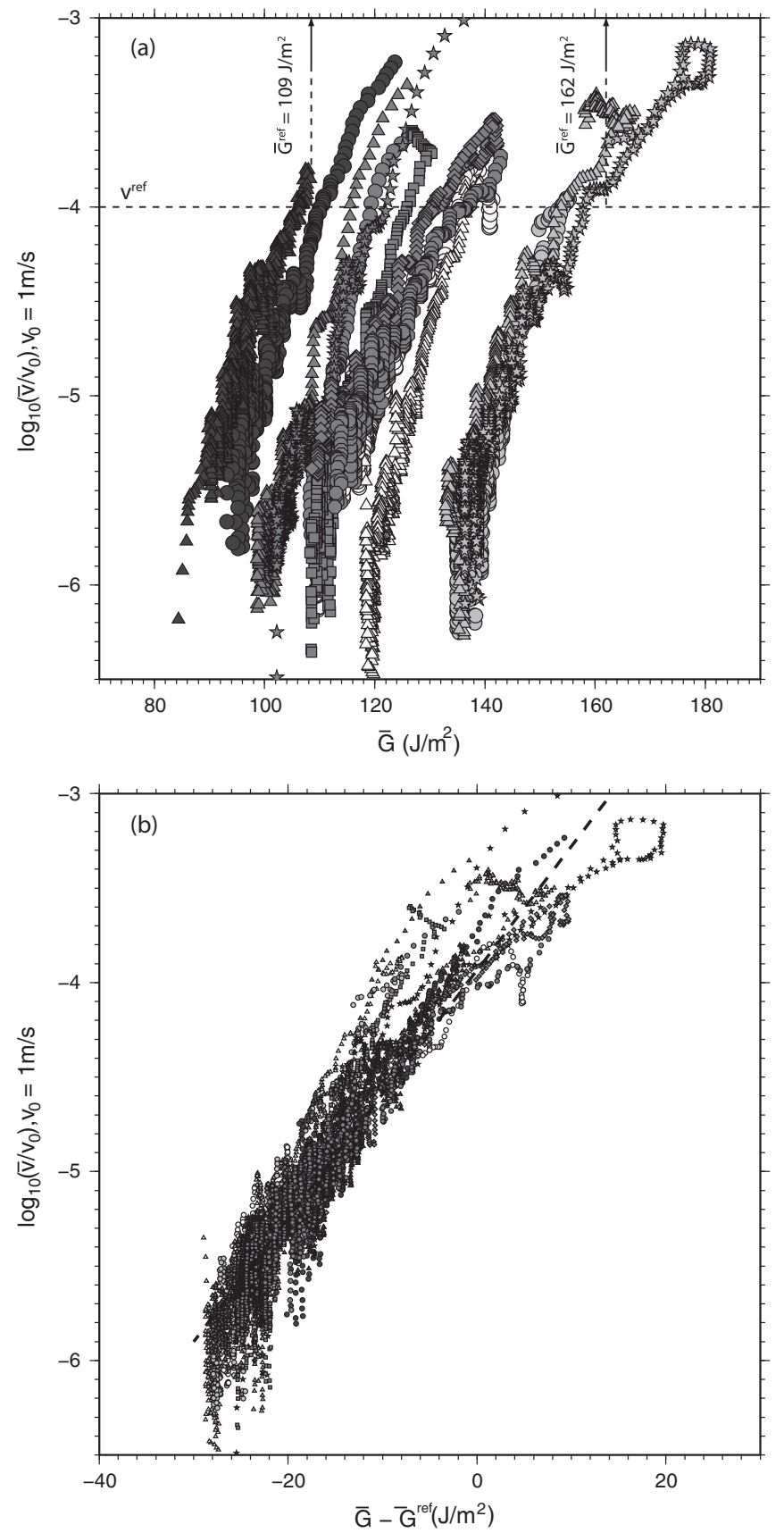

FIG. 6. (a) Variation of the logarithm of the average crack-front velocity as a function of the average energy release rate. All experiments are reported in this figure and are distinguished by gray levels and symbols. Similar gray levels indicate experiments performed in the same region of one specific plate (short separation distance between fracture onsets). The reference velocity $v_{\text {ref }}$ used to estimate $\bar{G}^{\text {ref }}$ is shown as a horizontal black line. Upper and lower bounds of $\bar{G}^{\text {ref }}$ are indicated with arrows. (b) Superimposition of all experiments when plotted as a function of $\bar{G}-\bar{G}^{\text {ref }}$. The dashed line represents the best-fitted model. The slope of this line is related to the value of $\alpha$ and is $\alpha^{2} / k_{B} T$.

[41,51]. Our experimental data do not allow us to discriminate among these different formulations. We consider the simple Arrhenius law introduced in Eq. (3), where the energy barrier is

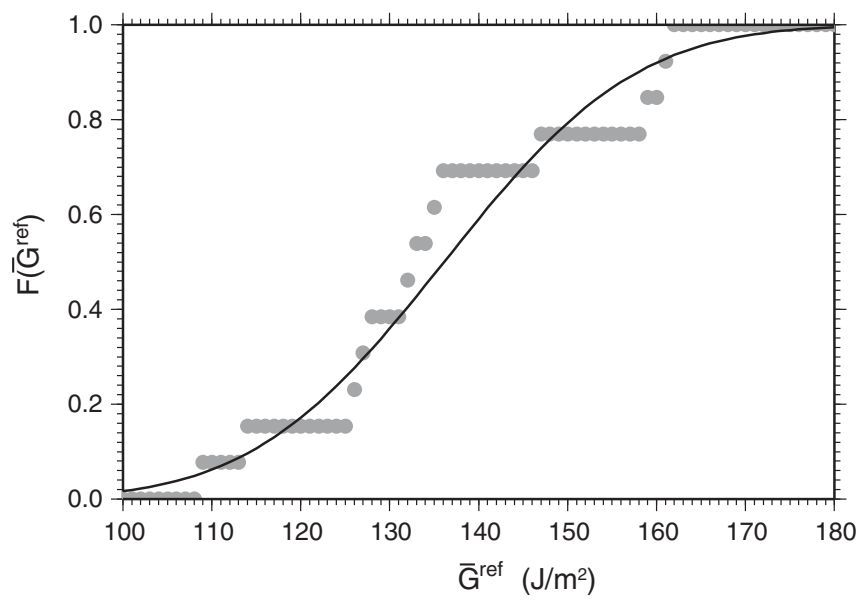

FIG. 7. Cumulative distribution function of the estimated values of $\bar{G}^{\text {ref }}$ for all experiments (gray dots). Values are distributed between 109 and $162 \mathrm{~J} / \mathrm{m}^{2}$ with a mean of $137 \mathrm{~J} / \mathrm{m}^{2}$. The dark curve represents the expected cumulative distribution function for a normal distribution with similar mean and standard deviation as inferred from our experimental data.

linearly related to the difference between $G$ and $G_{c}$, a relevant model of the fracture process for our experiments. Indeed, it fits our experimental data over several orders of magnitude of average crack speed (Fig. 6).

\section{NUMERICAL SOLUTIONS}

We solve the Arrhenius law [Eq. (3)] with the prescribed loading and dependence of $\bar{G}$ on $\bar{a}$ and $u$ [Eq. (8)] to obtain predictions of the evolution, for each experiment, of the front position, the loading force, and the energy release rate. This allows a second check on the validity of the activated energy mechanism to describe the crack-front propagation. Unfortunately, setting $u=V_{l} t$ or $u=$ const in Eq. (8) does not lead to analytical solutions. Therefore, we proceed to solve Eq. (8) numerically.

We first introduce a characteristic speed $v_{1}$ defined as

$$
\begin{aligned}
v_{1} & =\chi \exp \left(-\frac{\alpha^{2} \bar{G}_{c}}{k_{B} T}\right) \\
& =v_{\text {ref }} \exp \left(-\frac{\alpha^{2} \bar{G}^{\text {ref }}}{k_{B} T}\right) .
\end{aligned}
$$

Then, we introduce dimensionless variables $\bar{a}^{\prime}=\bar{a} / \lambda$ and $t^{\prime}=$ $t / \tau$ that transform Eq. (8) into a simpler form. In the forced regime we introduce

$$
\begin{gathered}
\lambda=\alpha \frac{V_{l}}{v_{1}}\left(\frac{3 E h^{3}}{8 k_{B} T}\right)^{1 / 2}, \\
\tau=\frac{\lambda}{v_{1}},
\end{gathered}
$$

which lead to

$$
\frac{d \bar{a}^{\prime}}{d t^{\prime}}=\exp \left(\frac{t^{\prime 2}}{\bar{a}^{\prime 4}}\right)
$$

where $u=V_{l} t$. 


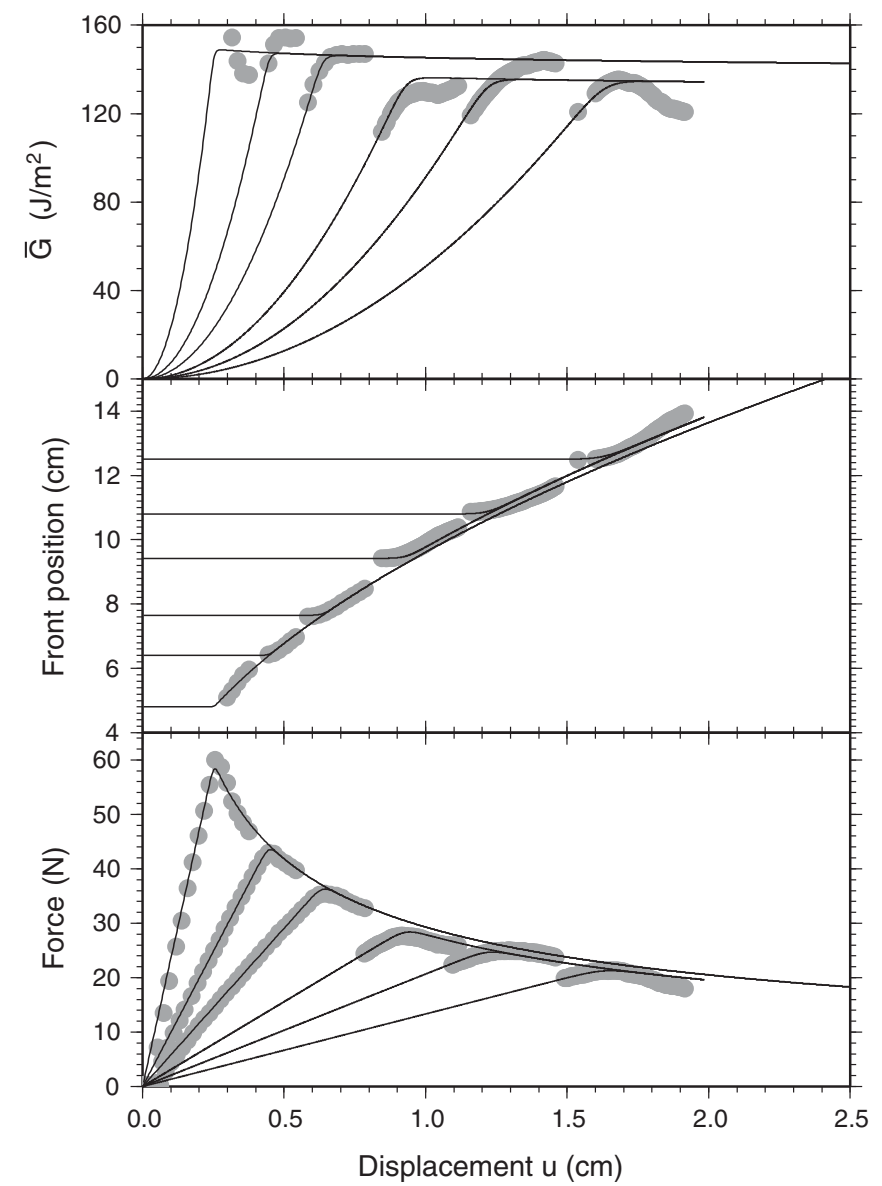

FIG. 8. Variation of force $F$, front position $\bar{a}$, and energy release rate $\bar{G}$ obtained during six experiments (gray points) carried out in the forced regime. The results of the numerical integration of Eq. (14) are drawn as black lines. The model captures most of the features observed in our experiments, such as the peak value of the force and its occurrence time. It also captures the appearance of a Griffith regime, i.e., the fact that $\bar{G}$ is close to constant at large enough crack speed.

We use Eq. (5) to compute the force from the front position. We numerically solve Eq. (14) using a simple Euler model with a constant time step. We found that the evolution of $\bar{a}$ and $F$ can both be well reproduced as solutions of Eq. (14) when we set $\alpha=2.5 \times 10^{-11} \mathrm{~m}$ and $\bar{G}^{\text {ref }}=152 \mathrm{~J} / \mathrm{m}^{2}$. These values fall in the range of estimated parameters (Fig. 7) and represent acceptable results. We should note that $\alpha$ and $\bar{G}^{\text {ref }}$ are not well constrained as they are highly correlated. A change in $\alpha$ can be balanced by a change in $\bar{G}^{\text {ref }}$. The evolution of force, front position, and $\bar{G}$ along with numerical solutions of Eq. (14) are shown in Fig. 8 for six experiments. The loading velocity is $V_{l}=310 \mu \mathrm{m} / \mathrm{s}$ for the first three experiments and $V_{l}=$ $62 \mu \mathrm{m} / \mathrm{s}$ for the remaining three experiments. The width of the plate is $2.84 \mathrm{~cm}$ and the Young modulus of the PMMA plate is $E=3.2 \mathrm{GPa}$. All solutions with a common loading velocity tend to align on the same curve after a sufficiently long time. This results from the common time dependence of Eq. (14) in all experiments with the same loading velocity. Differences arise from the variation of the initial front position at the start of the experiment. Our numerical model, resulting from the coupling of the cantilever beam configuration of our system and the activated energy mechanism necessary to describe the fracture process, is in good agreement with the evolution of the force, front position, and energy release rate (Fig. 8).

The integration of this thermal activation model with the large-scale elastic mechanics, over these characteristic time scales, with a constant velocity loading, leads to roughly constant values of $\bar{G}$ during the propagation: this means that the fact that $\bar{G}=$ constant, i.e., a Griffith regime, results from the direct integration of this subcritical model with a proper forcing in the evaluation of $\bar{G}$.

The numerical model depends on the choice of several parameters $(E, b, h)$ that are affected by uncertainties. It is possible to improve the fits to our data by varying these parameters within their uncertainty range. However, the discrepancy between the model and the data is small and could also be the result of second-order effects affecting the crack propagation (e.g., viscoelastic effects).

We now turn to the relaxation regime where the beam deflection is constant. We observe an evolution of the front position (Fig. 4) that is a priori not compatible with LEFM theory and the viscous rheology model as used in simulations, e.g., by Bonamy et al. [52], which assumed that the transition to zero propagation velocity of the front is sharp when the energy release rate is below the critical energy for fracture propagation (Griffith criterion, zero-temperature limit). We obtain the evolution of the crack front in this relaxation regime by setting $u_{\text {stop }}=V_{l} t_{\text {stop }}$ in Eq. (8) and solving the dimensionless differential equation:

$$
\begin{gathered}
\lambda=\left(\frac{3 E h^{3} \alpha^{2} u_{\text {stop }}^{2}}{8 k_{B} T}\right)^{1 / 4}, \\
\tau=\frac{\lambda}{v_{1}}
\end{gathered}
$$

leading to

$$
\frac{d \bar{a}^{\prime}}{d t^{\prime}}=\exp \left(\frac{1}{\bar{a}^{\prime 4}}\right) .
$$

We keep the same estimate of $\alpha$ and $\bar{G}^{\text {ref }}$, that we obtained in the constant-loading-velocity regime, when solving Eq. (17). We compare the results of the integration of Eq. (17) to the experimental observations shown in Fig. 2. The evolution of the force and front position obtained from the experimental data and from the numerical model are shown Fig. 9. The fit to the front position provided by the integration of Eq. (17) is in close agreement with the observations. We also fitted our numerical model to the long-lasting experiment (18 h of relaxation). As this experiment was performed on a different sample, we calculated $\bar{G}^{\text {ref }}$ and $\alpha$ for this experiment. We obtained our best fit by setting $\alpha=1.8 \times 10^{-11} \mathrm{~m}$ and $\bar{G}^{\text {ref }}=142 \mathrm{~J} / \mathrm{m}^{2}$ (Fig. 9). Both force and front position are well reproduced by the numerical model [dark continuous line in Figs. 9(a) and 9(b)]. However, the front position departs from the computed position at long times [Fig. 9(b)]. This may imply that additional processes are taking place and affecting the front propagation or that some parameters, considered as constant, did actually vary over the course of this experiment, for instance temperature or the broad scale of the fracture toughness. 


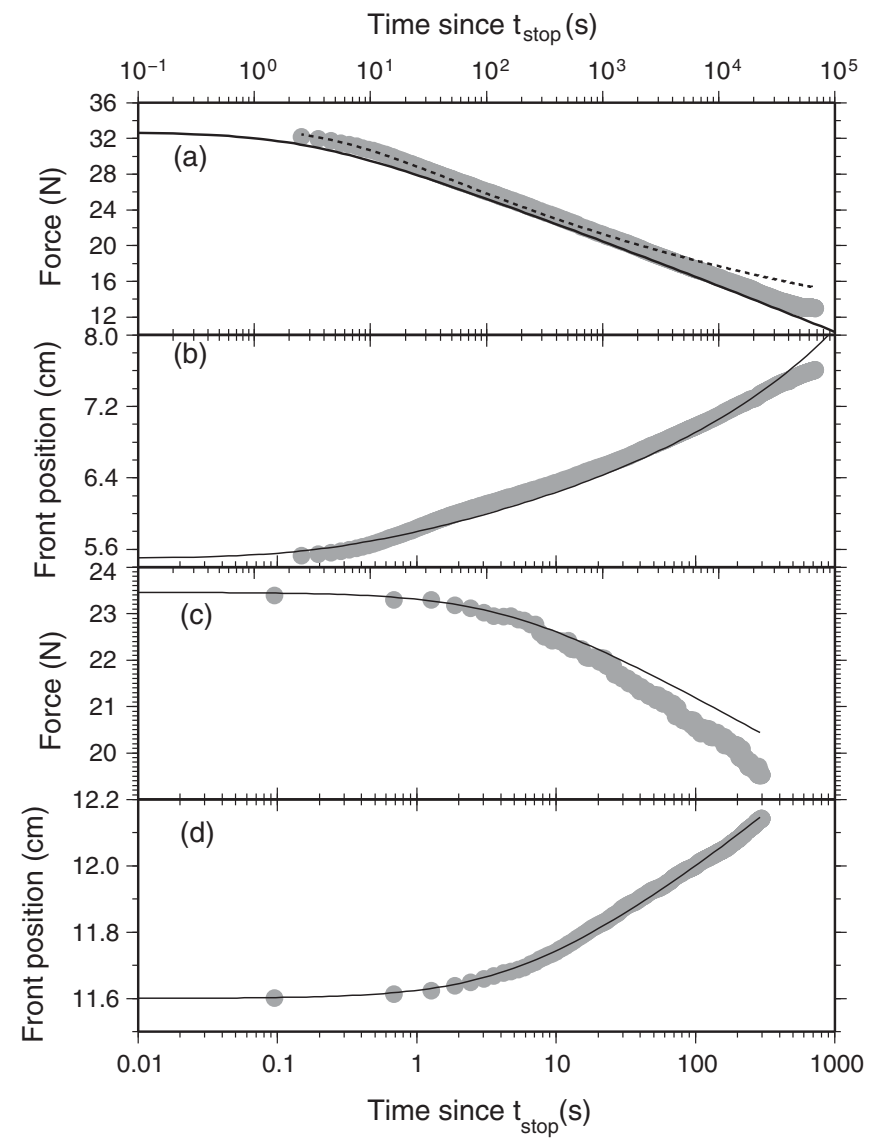

FIG. 9. Variation of force $F$ and front position $\bar{a}$ obtained during the relaxation regime for the experiment displayed on Fig. 2 and for 5 min [gray dots, (c) and (d)]. Results of the numerical integration are represented as black line. Same representation is made for the experiments monitored for more than $18 \mathrm{~h}$ in the relaxation regime and is displayed in the two upper graphs [(a) and (b)]. The dashed line shows the evolution of force deduced from the fit of the front position from Eq. (1).

\section{DISCUSSION}

Equation (8) governs the large-scale motion of the crack. Unfortunately, it has no analytical solutions for the two loading regimes of interest to our experiments. Nonetheless, we show that two simple approximations lead to analytical expressions of the front advance in close agreement with our experimental observations.

\section{A. Constant- $G$ approximation}

Here we focus on the case where the energy release rate can be considered as constant: $\bar{G}=$ const. This condition corresponds to a constant crack velocity $\bar{v}=$ const [Eq. (3)]. We clearly observe the propagation of the front reaching a quasiconstant velocity during the forced regime ( $V_{1}=$ const $\neq$ 0) after an initiation phase [Fig. 2(b)]. An initiation phase is expected as the front velocity cannot jump instantaneously from zero to its steady state value. To first order, the energy release rate $\bar{G}$ remains the same in the forced regime, $\bar{G}=G_{0}$, during the crack propagation.
For a constant energy release rate $G$, we derive from Eq. (7)

$$
\bar{G}=\frac{3 E \delta^{3}}{8} \frac{u^{2}}{\bar{a}^{4}}=G_{0}=\text { const. }
$$

Introducing $A=\left(\frac{3 E \delta^{3}}{8 G_{0}}\right)^{1 / 4}$, we get

$$
\bar{a}(u)=A u^{1 / 2} .
$$

Therefore a constant energy release rate implies a scaling between the average front position $\bar{a}$ and the deflection $u$. In fact, Eq. (19) is of the form of the fit used in Fig. 3. It thus provides a physical basis to the empirical relation proposed to fit the data.

On the other hand, the result of Eq. (19) implies that $\bar{v}(t) \propto V_{l} / \bar{a}$ as $u \propto V_{l} t$ in the forced regime. The front velocity decreases at constant $G$ as the crack front advances. However, as $\bar{a}$ is small around the initial front position for each incremental experiment along the same sample, $\bar{v}$ is small as well. Finally, it is noticeable in Fig. 8 that the experimental evolution of the energy release rate $\bar{G}$ is almost constant for the entire duration of the investigated range after an initiation period well reproduced by the complete numerical integration of the Arrhenius law [Eq. (8)]. This is also in agreement with the hypothesis of $\bar{G}=$ const in this forced regime.

\section{B. First-order approximation of $\boldsymbol{G}$}

Now we focus on characterizing the relaxation process $(t>$ $\left.t_{\text {stop }}\right)$. We recall that the loading velocity is zero $\left(V_{l}=0\right.$ for $t>$ $\left.t_{\text {stop }}\right)$ during this time interval. The force decreases with time while the average position of the front continues to increase in a logarithmic fashion (Figs. 2 and 4). We analyze a first-order approximation of the solution assuming that $\bar{a}(t)$ is small in this relaxation regime. We derive a first-order expansion of $\bar{G}$ around some position $\bar{a}_{0}=\bar{a}\left(t_{\text {stop }}\right)$ :

$$
\bar{G}=G_{0}+\frac{d \bar{G}\left(\bar{a}_{0}\right)}{d \bar{a}}\left(\bar{a}-\bar{a}_{0}\right),
$$

where $G_{0}=\bar{G}\left(\bar{a}_{0}\right)$ and $\frac{d \bar{G}\left(\bar{a}_{0}\right)}{d \bar{a}}$ can be computed from Eq. (7) as

$$
\frac{d \bar{G}\left(\bar{a}_{0}\right)}{d \bar{a}}=-\frac{4 G_{0}}{\bar{a}_{0}} .
$$

Replacing (20) and (21) into Eq. (3), we obtain

$$
\bar{v}=\chi^{\prime} \exp \left(-\frac{4 \alpha^{2} G_{0} \bar{a}}{\bar{a}_{0} k_{B} T}\right)
$$

with $\chi^{\prime}=\chi \exp \left(\alpha^{2} \frac{-\bar{G}_{c}+5 G_{0}}{k_{B} T}\right)$. The above equation integrates to

$$
\bar{a}(t)=\frac{k_{B} T \bar{a}_{0}}{4 \alpha^{2} G_{0}} \ln \left[\frac{4 \chi^{\prime} G_{0} \alpha^{2}}{k_{B} T \bar{a}_{0}}\left(t-t_{0}\right)+\exp \left(\frac{4 G_{0} \alpha^{2}}{k_{B} T}\right)\right],
$$

where $t_{0}$ and $\bar{a}_{0}$ are the lower integration limits, corresponding to the initial time and position, respectively, where we consider the creep conditions to apply. The above expression can be simplified by setting $H_{0}=\frac{\left(5 G_{0}-\bar{G}_{c}\right) \alpha^{2}}{k_{B} T}, \beta=\frac{5 G_{0}-\bar{G}_{c}}{4 G_{0}}$, and 
introducing the characteristic time $t^{*}=\frac{\beta \bar{a}_{0}}{H_{0} \chi} \exp \left[-H_{0}(1-\right.$ $1 / \beta)]$. This yields

$$
\bar{a}(t)-\bar{a}_{0}=\frac{\bar{a}_{0} \beta}{H_{0}} \ln \left[\frac{t-t_{0}}{t^{*}}+1\right] .
$$

At large times, when $t-t_{0} \gg t^{*}$, the above equation reduces to

$$
\begin{aligned}
\bar{a}(t)-\bar{a}_{0} & \approx \frac{\bar{a}_{0} \beta}{H_{0}} \ln \left(\frac{t-t_{0}}{t^{*}}\right), \\
\bar{v}(t) & \approx \frac{\bar{a}_{0} \beta}{H_{0}} \frac{1}{t-t_{0}} .
\end{aligned}
$$

Logarithmic relaxation has also been observed in granular media [21]. In rock mechanics, Scholz [16] developed a simple model of creep in heterogeneous media where the collective behavior resulting from local decreases of strength leads to a similar $\propto t^{-1}$ evolution of the deformation rate during the creep regime. The functional form of Eq. (24) is similar to that of Eq. (1) used to describe the evolution of the front in this relaxation regime (Fig. 4). It implies that Eq. (24) also provides a good description of the data and that our firstorder approximation is valid in the range investigated in our experiments.

\section{CONCLUSIONS}

We explored the average velocity of an interfacial crack under two different loading conditions. These are (1) a forced regime with imposed constant loading velocity and (2) a relaxation regime. Both conditions induce crack velocities much lower than the Rayleigh wave velocity of the material. As often observed for homogeneous or weakly heterogeneous materials, the constant-speed loading regime is compatible with a constant energy release rate, and the relaxation regime is compatible with a logarithmic deformation. The macroscopic evolution of the crack front is smooth and continuous, characteristic of creep processes. This is opposite to what is observed and modeled at the local scale, namely, an intermittency of the fracture process described by a succession of local discrete brittle failures [8,30]. Such "brittle-creep" behavior has been extensively studied in rocks where acoustic emissions are recorded during the otherwise slow global deformation of the sample (e.g., [16,41,53]) or in other materials like paper $[12,42,54]$.

We developed a thermally activated fracture model that consists of a set of independent microscopic sites that break according to an Arrhenius law. The energy barrier is assumed to be a function of the difference between the local energy release rate $G$ and the local critical energy release rate $G_{c}$. We show for independent microscopic sites in time and space that the model reduces to $1 \mathrm{D}$ where the crack tip advance is controlled by the difference between the average energy release rate $\bar{G}$ and the average critical energy release rate $\bar{G}_{c}$. The model describes the fracturing process in the subcritical regime as a mechanism sensitive only to the applied stress on the crack tip and the effective toughness of the material. It allows the crack propagation to be driven below the critical energy release rate by statistical stress fluctuations that trigger rupture at the scale of atomic bonds. The typical length scale of the process is on the order of picometers, in agreement with values reported by
Ponson [41] for rock samples and by Santucci et al. [42] for paper. This suggests that the creeping mechanism we observe is related to breakage at the typical length scale of atomic bonds and that stress fluctuations can trigger rupture of these atomic bonds. The length scale of this process, a few $10^{-11}-10^{-10}$ $\mathrm{m}$, is much smaller than the correlation length scale of the toughness heterogeneity of the material, typically on the order of the glass bead size used during sample preparation blasting $\left(1 \times 10^{-4}-5 \times 10^{-4} \mathrm{~m}\right)[50,55]$.

All experimental data of the macroscopic evolution of the crack are well explained by the proposed subcritical mechanism despite the different tested loading conditions. Neglect of this temperature effect does not give a correct description of the crack propagation in a constant-deflection regime during relaxation tests. Moreover, several factors that would possibly influence the progression of the crack have been disregarded. They include possible finite mechanical rotation of the plate, viscoelastic flows inside the bulk of the PMMA plate like microbubbles, or chemical processes. The thermally activated model we propose clearly reproduces the evolution of the front during experiments over a large range of time scales (from $10^{-1}$ to more than $10^{4} \mathrm{~s}$ ) and different loading paths, at least when the average advance of the front, $\bar{a}$, is small compared to its initial position. Therefore, neglected mechanisms have second-order effects on the crack evolution compared to the stress-induced creeping mechanism.

We reproduce the large-scale evolution of the front propagation despite a simple description of the heterogeneous nature of the material. The macroscopic behavior of the interface with spatially fluctuating toughness induced by the sandblasting procedure is represented by an average quantity. This might be due to the limited heterogeneity of the toughness when seen at a centimeter scale, as evidenced by the almost flat geometry of the crack front line (we never observed crack-front distortions bigger than $10 \%$ of the system scale). Although heterogeneities are present and are encountered during the crack propagation, the macroscopic scale evolution is well described by the average energy release rate, an average quantity among all local sites. Such large-scale information appears sufficient to predict the overall large-scale dynamics of the propagating crack under various loading regimes.

\section{ACKNOWLEDGMENTS}

We acknowledge Alain Steyer for technical support. The project was supported by a French ANR RiskNat SUPNAF grant, by a CNRS French-Norwegian PICS, and by the INSU CESSUR program. J.E.E. was supported by the Caltech Seismological Laboratory. K.J.M. was partly supported by a visiting grant from Strasbourg University.

\section{APPENDIX}

In our configuration, the force applied on the plates is mostly tangential to the plates, which contributes to reduce mode II loading: this is achieved by applying the force at the free end of the bent plate with a freely rotating rod (using ball bearings). Furthermore, the rod is made of polyamide PA 6.6 (nylon) and thus has a low friction coefficient. It ensures that minimum shear forces are actually applied on the bending plate 


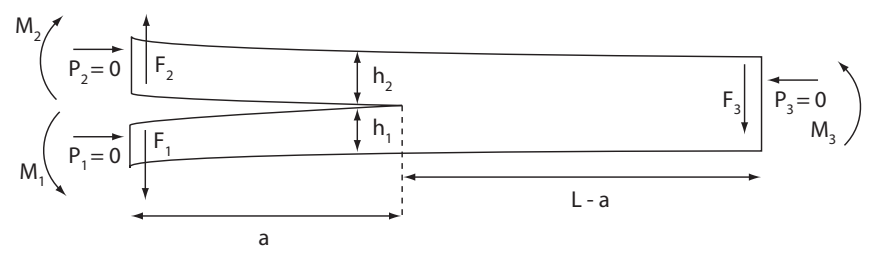

FIG. 10. General configuration of bent plates, adapted from [56] to the configuration used in this work.

and thus limits the existence of mode II fracture component in our experiments.

Even though this tangential force at the free end of the beam is reduced, the loading asymmetry in our configuration, and the unequal thickness of the two plates, may induce some mode II component at the crack tip. This can be precisely evaluated by following the development detailed below. Following the general formulation presented in [56] for a nonsymmetric beam configuration, based on the evaluation of the $J$ integral in a general two-plate configuration, we can calculate precisely the values of the forces per unit length applied on the ends of the plates both normal to the plates, $F_{1}, F_{2}$, and $F_{3}$, and tangential, $P_{1}, P_{2}$, and $P_{3}$, as well as the torques per unit length with respect to the tip, $M_{1}, M_{2}$, and $M_{3}$; see Fig. 10 .

The design of the setup guarantees that the tangential components are minimal with respect to the normal ones: we will consider that $P_{1}=P_{2}=P_{3}=0$. The distance between the tip and the rod being denoted $\bar{a}$, and $F_{l}=F / b$ the force exerted on the lower plate ( $F$ is the total force, and $b$ the plate width; hence the force per unit length is $F_{l}=F / b$ ), one writes $F_{1}=F_{l}$, and the torque on the lower plate is $M=M_{1}=F_{l} \bar{a}$. The torques on the upper plate and on the opposite end are $M_{2}=F_{2} \bar{a}$ and $M_{3}=F_{3}(L-\bar{a})$, where $L$ is the plate length. The equilibrium between the forces ensures that $F_{1}=F_{2}-F_{3}$, and the one between the torques (nonrotation of the plates) that $M_{1}=M_{2}+M_{3}$. Hence, we have

$$
\begin{gathered}
F_{2}-F_{3}=F_{l}, \\
F_{2} \bar{a}+F_{3}(L-\bar{a})=F_{l} \bar{a},
\end{gathered}
$$

which leads to

$$
F_{3}=0, \quad F_{2}=F_{1}=F_{l}, \quad M_{2}=M_{1}=M=F_{l} \bar{a} .
$$

From Eq. (3.7) in Hutchinson and Suo [56] this allows us to obtain separately the values of the stress intensity factors $K_{I}$ and $K_{I I}$ in order to evaluate their relative importance. From the general asymmetric beam configuration and applying the particular boundary condition imposed in our system, we get with the thicknesses of the two plates, $h_{1}=0.5 \mathrm{~cm}$ and $h_{2}=$ $1 \mathrm{~cm}$, the ratio $\eta=h_{1} / h_{2}=0.5 / 1=0.5$, and the constants, evaluated from [56],

$$
\begin{gathered}
\sin (\gamma)=(6 \sqrt{U V}) \eta^{2}(1+\eta), \\
1 / V=12\left(1+\eta^{3}\right), \\
1 / U=1+4 \eta+6 \eta^{2}+3 \eta^{3}, \\
\omega=52.1^{\circ}-3^{\circ} \eta,
\end{gathered}
$$

which leads to

$$
V=0.074
$$

$$
\begin{aligned}
U & =0.21, \\
\gamma & =16^{\circ}, \\
\omega & =51^{\circ} .
\end{aligned}
$$

With these constants, the general formula Eq. (3.7) in [56] reduces to

$$
\begin{gathered}
K_{I}=\frac{\bar{a} F_{l}}{\sqrt{2 h_{1}^{3} V}} \sin (\omega+\gamma), \\
K_{I I}=-\frac{\bar{a} F_{l}}{\sqrt{2 h_{1}^{3} V}} \cos (\omega+\gamma) .
\end{gathered}
$$

This yields an estimate of

$$
K_{I I} / K_{I}=0.43 .
$$

It thus implies that the mode II component is not negligible in our problem. Taking into account the details of this asymmetry leads to the formulation of the energy release rate $\bar{G}$ for a propagation of the crack at the interface between the two plates in our system, in mixed mode. The energy release rate does not separate the rupture modes, which present a similar dependence on $\bar{a}$. The fact that the fracture propagates along the interface is due to a combination of factors: First, $K_{I I}$ remains smaller than $K_{I}$, which does not favor an out-of-plane branching of the crack. Next, the toughness $K_{c}$ is smaller along the interface than anywhere else in the bulk of the plates, due to the preparation of the samples, for both modes I and II. It thus favors a propagation of the crack along the interface, as is indeed observed.

Following the general formulation from [56] [or directly from their Eq. (3.6)], the energy release rate per unit length in our system is thus

$$
\begin{aligned}
\bar{G} & =\bar{G}_{I}+\bar{G}_{I I} \\
& =\left(K_{I}^{2}+K_{I I}^{2}\right) / E \\
& =\frac{M^{2}}{2 E h_{1}^{3} V}\left[\sin ^{2}(\omega+\gamma)+\cos ^{2}(\omega+\gamma)\right] \\
& =\frac{F_{l}^{2} \bar{a}^{2}}{2 E h_{1}^{3}}\left[12\left(1+h_{2}^{2} / h_{1}^{2}\right)\right] \\
& =\frac{6 F_{l}^{2} \bar{a}^{2}}{E h^{3}},
\end{aligned}
$$

where $h^{3}$ is defined as

$$
\frac{1}{h^{3}}=\frac{1}{h_{1}^{3}}+\frac{1}{h_{2}^{3}}
$$

and $h_{1}$ and $h_{2}$ are respectively the thicknesses of the bottom and the upper plate. This then yields

$$
G=\frac{6 F^{2} \bar{a}^{2}}{E h^{3} b^{2}}=\frac{1}{b} \frac{d U_{E}}{d \bar{a}}
$$

which has the opposite sign to Eq. (4) because

$$
\left.\frac{d U_{E}(F)}{d \bar{a}}\right|_{F}=-\left.\frac{d U_{E}(u)}{d \bar{a}}\right|_{u}
$$

[4]. Integration of the previous expression shows that

$$
U_{E}=\frac{2 F^{2} \bar{a}^{3}}{E b h^{3}},
$$


and since due to linear elasticity, the stored elastic energy is

$$
U_{E}=F u / 2 \text {, }
$$

it leads to

$$
u=\frac{4 F \bar{a}^{3}}{E h^{3} b}, \quad F=\frac{E h^{3} b u}{4 \bar{a}^{3}},
$$

so that

$$
\bar{G}=\frac{3 u^{2} E h^{3}}{8 \bar{a}^{4}} .
$$

If one used instead an estimate of $\bar{G}$ based on a pure mode I, symmetric loading hypothesis, one would get

$$
\bar{G}=\frac{3 u^{2} E h_{1}^{3}}{8 \bar{a}^{4}} .
$$

Taking into account this asymmetry thus leads to a change in the estimate of $\bar{G}$ by a factor

$$
\rho=\frac{h_{1}^{3}+h_{2}^{3}}{h_{2}^{3}} .
$$

As $h_{1}=5 \mathrm{~mm}$ and $h_{2}=1 \mathrm{~cm}$ in our experiments, it then yields $\rho=1.125$.
[1] B. K. Atkinson, Fracture Mechanics of Rocks (Academic Press, San Diego, 1991).

[2] E. Schulson and P. Duval, Creep and Fracture of Ice (Cambridge University Press, Cambridge, 2009).

[3] I. Smith, E. Landis, and M. Gong, Fracture and Fatigue in Wood (John Wiley \& Sons, New York, 2003).

[4] T. L. Anderson, Fracture Mechanics-Fundamentals and Applications, 2nd ed. (CRC Press, Boca Raton, FL, 1995).

[5] B. Lawn, Fracture of Brittle Solids, 2nd ed. (Cambridge University Press, Cambridge, 1993).

[6] L. Freund, Dynamic Fracture Mechanics (Cambridge University Press, Cambridge, 1990).

[7] J. Fineberg and M. Marder, Phys. Rep. 313, 1 (1999).

[8] D. Bonamy, J. Phys. D 42, 214014 (2009).

[9] D. Unger, Analytical Fracture Mechanics (Dover, New York, 1995).

[10] J. R. Rice, J. Mech. Phys. Solids 26, 61 (1978).

[11] L. D. Landau and Lifshitz, Statistical Physics, Part 1, 3rd ed. (Pergamon, Oxford, 1980).

[12] L. Vanel, S. Ciliberto, P.-P. Cortet, and S. Santucci, J. Phys. D 42, 214007 (2009).

[13] S. W. Freiman, J. Geophys. Res. 86, 4072 (1984).

[14] S. M. Wiederhorn and L. H. Bolz, J. Am. Ceram. Soc. 53, 543 (1970).

[15] R. J. Charles, J. Appl. Phys. 29, 1549 (1958).

[16] C. H. Scholz, J. Geophys. Res. 73, 3295 (1968).

[17] E. Favier, V. Lazarus, and J.-B. Leblond, in Modern Practice in Stress and Vibration Analysis 2003 (MPSVA2003), Glasgow, edited by M. S. Forum (Trans Tech Publications, Zurich, 2003), Vol. 440-441, pp. 153-160.

[18] N. Pindra, V. Lazarus, and J.-B. Leblond, J. Mech. Phys. Solids 58, 281 (2010).

[19] V. Lazarus, J. Mech. Phys. Solids 59, 121 (2011).

[20] S. N. Zhurkov, Int. J. Fract. Mech. 1, 311 (1965).

[21] R. R. Hartley and R. P. Behringer, Nature (London) 421, 928 (2003).

[22] L. Bocquet, E. Charlaix, S. Ciliberto, and J. Crassous, Nature (London) 396, 735 (1998).

[23] G. Løvoll, K. J. Måløy, and E. G. Flekkøy, Phys. Rev. E 60, 5872 (1999).

[24] M. Sahimi, Heterogeneous Materials II: Nonlinear and Breakdown Properties and Atomistic Modeling (Springer-Verlag, New York, 2003).
[25] W. Weibull, J. Appl. Mech. 18, 293 (1951).

[26] Z. Bažant, Scaling of Structural Strength, 2nd ed. (ButterworthHeinemann, London, 2005).

[27] J. Obreimoff, Proc. R. Soc. London 127, 290 (1930).

[28] J. Schmittbuhl and K. J. Måløy, Phys. Rev. Lett. 78, 3888 (1997).

[29] A. Delaplace, J. Schmittbuhl, and K. J. Måløy, Phys. Rev. E 60, 1337 (1999).

[30] K. J. Måløy, S. Santucci, J. Schmittbuhl, and R. Toussaint, Phys. Rev. Lett. 96, 045501 (2006).

[31] K. T. Tallakstad, R. Toussaint, S. Santucci, J. Schmittbuhl, and K. J. Måløy, Phys. Rev. E 83, 046108 (2011).

[32] H. Gao and J. R. Rice, J. Appl. Mech. 56, 828 (1989).

[33] J. Schmittbuhl, S. Roux, J.-P. Vilotte, and K. J. Måløy, Phys. Rev. Lett. 74, 1787 (1995).

[34] S. Ramanathan and D. S. Fisher, Phys. Rev. B 58, 6026 (1998).

[35] S. R. Pride and R. Toussaint, Physica A 312, 159 (2002).

[36] R. Toussaint and S. R. Pride, Phys. Rev. E 71, 046127 (2005).

[37] J. Schmittbuhl, A. Hansen, and G. G. Batrouni, Phys. Rev. Lett. 90, 045505 (2003).

[38] M. Alava, P. Nukalaz, and S. Zapperi, Adv. Phys. 55, 349 (2006).

[39] S. Pradhan, A. Hansen, and B. Chakrabarti, Rev. Mod. Phys. 82, 499 (2010).

[40] M. Grob, J. Schmittbuhl, R. Toussaint, L. Rivera, S. Santucci, and K. J. Måløy, Pure Appl. Geophys. 166, 777 (2009).

[41] L. Ponson, Phys. Rev. Lett. 103, 055501 (2009).

[42] S. Santucci, L. Vanel, and S. Ciliberto, Phys. Rev. Lett. 93, 095505 (2004).

[43] A. Saichev and D. Sornette, Phys. Rev. E 71, 016608 (2005).

[44] P. Cortet, L. Vanel, and S. Ciliberto, Europhys. Lett. 74, 602 (2006).

[45] Z. Bažant and S. Pang, J. Mech. Phys. Solids 55, 91 (2007).

[46] R. Toussaint and S. R. Pride, Phys. Rev. E 66, 036135 (2002).

[47] R. Toussaint and S. R. Pride, Phys. Rev. E 66, 036136 (2002).

[48] R. Toussaint and S. R. Pride, Phys. Rev. E 66, 036137 (2002).

[49] R. Toussaint and A. Hansen, Phys. Rev. E 73, 046103 (2006).

[50] O. Lengliné, J. Schmittbuhl, J. Elkhoury, J.-P. Ampuero, R. Toussaint, and K. J. Måløy, J. Geophys. Res. 116, B08215 (2011).

[51] A. B. Kolton, A. Rosso, and T. Giamarchi, Phys. Rev. Lett. 94, 047002 (2005).

[52] D. Bonamy, S. Santucci, and L. Ponson, Phys. Rev. Lett. 101, 045501 (2008). 
[53] B. K. Atkinson, J. Geophys. Res. 89, 4077 (1984).

[54] S. Santucci, L. Vanel, and S. Ciliberto, Eur. Phys. J Spec. Top. 146, 341 (2007).

[55] S. Santucci, K. J. Måløy, R. Toussaint, and J. Schmittbuhl, in Dynamics of Complex Interconnected Systems: Networks and
Bioprocesses, edited by A. T. Skjeltorp and A. V. Belushkin, NATO Science Series II: Mathematics, Physics and Chemistry Vol. 232 (Springer, Dordrecht, 2006), pp. 49-59.

[56] J. W. Hutchinson and Z. Suo, Advances in Applied Mechanics (Academic Press, New York, 1992), Vol. 29, pp. 63-191. 